Muñoz, J.J., On the modelling of incompressibility in linear and non-linear elasticity with the master-slave approach, International Journal for Numerical Methods in Engineering, Vol. 74, Issue 2, pp. 269-293, 2008

\title{
On the modelling of incompressibility in linear and non-linear elasticity with the master-slave approach
}

\author{
J J Muñoz ${ }^{1, *}$ \\ 1 LaCàN, Dep. of Applied Mathematics III, Univ. Pol. Catalunya, Barcelona. Spain
}

\begin{abstract}
SUMMARY
The master-slave approach is adapted to model the kinematic constraints encountered in incompressibility. The method presented here allows to obtain discrete displacement and pressure fields for arbitrary finite element formulations that have discontinuous pressure interpolations. The resulting displacements satisfy exactly the incompressibility constraints in a weak sense, and are obtained solving a system of equations with the minimum (independent) degrees of freedom. In linear analysis, the method reproduces the well known stability results for inf-sup compliant elements, and permits to compute the pressure modes (physical or spurious) when they exist. By rewriting the equilibrium equations of a hyperelastic material, the method is extended to non-linear elasticity, while retaining the exact fulfilment of the incompressibility constraints in a weak sense. Problems with analytical solution in two and three dimensions are tested and compared to other solution methods. Copyright (c) 2006 John Wiley \& Sons, Ltd.
\end{abstract}

KEY WORDS: Master-slave, constraints, incompressibility, inf-sup, mixed elements, hyperelasticity

*Correspondence to: j.munoz@upc.edu 


\section{INTRODUCTION}

Robust and stable computational methods for incompressibility are crucial for the proper understanding of Newtonian incompressible fluids or rubber-like materials. In linear analysis, both applications are governed by equivalent equations (see for instance [18]), but for nonlinear analysis, the two problems lead to rather different expressions. In the present paper we will concentrate our study to the latter case: elastic incompressible problems, linear and nonlinear. Non-linear incompressible analyses have gained special attention during the last decade, partly due to its application in metal plasticity, soil mechanics or more recently, biomechanics.

On the other hand, research on the modelling of incompressibility has been carried out for more than forty years. The stability and solvability conditions of the resulting equations are now well understood in linear analysis. It has been shown that the existence, uniqueness and stability of the solution requires the fulfilment of the Ladyzhenskaya-Brezzi-Babuska (LBB) or inf-sup condition (see for instance [7, 12, 24]), which is satisfied by only certain pairs of interpolated displacements (or velocities) and pressures. However, discretisations that satisfy the LBB condition may become computationally expensive (the low order Q1P0 element is not LBB-compliant). For this reason, it is desirable to either stabilise the discretisations that do not satisfy the LBB condition by adding additional terms in the weak form (see for instance the early works $[14,25])$, or to provide efficient algorithms for those discretisations that satisfy the LBB condition $[1,24,13]$. The present paper studies the latter case and extends the solution of mixed formulations to non-linear elasticity interpolated with general pairs of displacementpressure interpolations. Stability predictions are not available for non-linear elasticity, and only results on the linearised problem or simple numerical examples exist [4, 35]. Moreover, it has been also demonstrated that in linear elasticity, some of the elements not satisfying the 
LBB condition can provide acceptable solutions for certain boundary conditions or applied body forces [6]. The mater-slave approach presented here becomes a useful tool to determine numerically whether the applied boundary conditions can lead to a unstable solution for general discretisations.

The master-slave approach has been initially designed for the modelling of the constraints in mechanisms $[28,26,30]$. However, resorting to similar ideas, we show that we can turn the equations of incompressible elasticity, discretised with mixed finite elements, into a displacement based system of equations which has the minimal number of degrees of freedom (dof). In fact, the master-slave approach provides a particular form of the null space method $[8,9]$ (or orthogonal projection), as it has been already applied in multibody systems dynamics $[29,30]$.

Projection techniques in linear incompressibility have been reported before [22]. However, in our approach, after using the constraint equations, we eliminate redundant displacement dof. This is tantamount to projecting the nodal displacements onto a discrete solenoidal displacement field, which we achieve in a global manner. Other techniques are based on the construction of elemental solenoidal fields, such as the Taylor-Hood element [36], the mini element [2], or the Crouzeix-Raviart element [17]. However, they require the enhancement of the discrete displacement filed by additional spaces. We remark that our method does not use any problem dependent parameters, in contrast to the penalty approaches or the also related mean dilatation method [10], or the UZAWA's algorithm [3, 12, 35].

It must be added though, that the global projection has a computational cost, namely the inversion of an additional matrix whose dimension is equal to the number of pressure degrees of freedom, and some additional global matrix multiplication. However, due to the fact that 
the resulting system of equations is considerably reduced, and that these additional operations are easily parallelisable, the overload of the method can be partly compensated, as it will be detailed.

In the master-slave approach presented here, the computation of the hydrostatic pressures is performed as a post-processing of the converged solution, and thus, in non-linear analysis, they do not need to be computed iteratively. Additionally, a set of spurious pressure modes may arise if (i) the boundary is fully prescribed with Dirichlet conditions, or (ii) non LBBcompliant mixed elements are used. While the former case is physically consistent (the pressure is determinated up to a constant value), in the latter case the pressure modes are a byproduct of the numerical discretisation employed. For both cases, the method presented here allows to compute the pressure modes, in linear and non-linear analysis.

For clarity in the exposition and in order to relate the method to other existent solution techniques for mixed formulations, we will first derive the discretised form arising from fully incompressible linear elasticity in Section 2. In Section 3 we present the master-slave approach for linear incompressible problems and, in Section 4, we introduced the extension to non-linear problems. Relevant numerical examples in two and three dimensions are obtained in Section 5 , and contrasted against their analytical solution.

\section{MIXED-FORMULATION FOR INCOMPRESSIBLE ELASTICITY}

\subsection{Continuous problem}

Let us first cast the equilibrium equations of a compressible linear elastic domain $\Omega \subset \mathbb{R}^{n_{s d}}$, with $n_{s d}$ the number of space dimensions. Given a displacement field $\boldsymbol{u}(\boldsymbol{x})$ at each point 
$\boldsymbol{x} \in \Omega$, the linear strain tensor is expressible as $\boldsymbol{\varepsilon}(\boldsymbol{u})=\frac{1}{2}\left(\nabla \boldsymbol{u}+\nabla \boldsymbol{u}^{\mathrm{T}}\right)=\frac{\varepsilon_{V}(\boldsymbol{u})}{3} \mathbf{I}+\bar{\varepsilon}(\boldsymbol{u})$, with $\varepsilon_{V}(\boldsymbol{u})=\operatorname{trace}(\varepsilon(\boldsymbol{u}))=\operatorname{div}(\boldsymbol{u})$ and $\bar{\varepsilon}(\boldsymbol{u})=\boldsymbol{\varepsilon}(\boldsymbol{u})-\frac{\varepsilon_{V}(\boldsymbol{u})}{3} \mathbf{I}$ its volumetric and deviatoric part, respectively. For incompressible materials, no volumetric deformation exists, and thus $\operatorname{div}(\boldsymbol{u})=0$. Let us assume that a surface load $\boldsymbol{g}$ is applied on the boundary $\Gamma_{g}$ and a prescribed displacement $\boldsymbol{u}_{0}$ on $\Gamma_{u}$, with $\partial \Omega=\Gamma_{g} \cup \Gamma_{u}$ and $\Gamma_{g} \cap \Gamma_{u}=\emptyset$. The equilibrium equations can be then posed as follows (see for instance $[12,18]$ ): Find $\boldsymbol{u} \in \mathcal{U}$ and $p \in \mathcal{Q}$ such that,

$$
\begin{array}{rlrl}
a(\boldsymbol{u}, \boldsymbol{v})-b(p, \boldsymbol{v}) & =(\boldsymbol{g}, \boldsymbol{v}), & & \forall \boldsymbol{v} \in \mathcal{V} \\
b(q, \boldsymbol{u}) & =0, & \forall q \in \mathcal{Q}
\end{array}
$$

where $a($,$) and b($,$) are bilinear forms associated to the deviatoric strain-energy and the$ incompressible condition, respectively, and $($,$) is a inner product (force term) associated to$ the external load $\boldsymbol{g}$. Their explicit expressions read:

$$
\begin{aligned}
a(\boldsymbol{u}, \boldsymbol{v}) & =2 \mu \int_{\Omega} \overline{\boldsymbol{\varepsilon}}(\boldsymbol{u}): \overline{\boldsymbol{\varepsilon}}(\boldsymbol{v}) d \Omega \\
b(p, \boldsymbol{v}) & =\int_{\Omega} p \operatorname{div} \boldsymbol{v} d \Omega \\
(\boldsymbol{g}, \boldsymbol{v}) & =\int_{\Omega} \boldsymbol{g} \cdot \boldsymbol{v} d \Omega,
\end{aligned}
$$

where $\mu$ is the shear modulus. Equation (1b) is the weak form of the local incompressibility condition $\varepsilon_{V}(\boldsymbol{u})=\operatorname{div}(\boldsymbol{u})=0, \forall \boldsymbol{x} \in \Omega$, and $p$ is the hydrostatic pressure. The space $\mathcal{Q}$ of pressures is required to be square-integrable, i.e. $\mathcal{Q}:=L^{2}$, and the space $\mathcal{U}$ of the trial functions is given by $\mathcal{U}=\left\{\boldsymbol{u} \in \mathcal{H}^{1}(\Omega)|\boldsymbol{u}|_{\Gamma_{u}}=\boldsymbol{u}_{0}\right\}$, where $\mathcal{H}^{1}$ is the Sobolev space of square- 
integrable functions with integrable first derivatives ${ }^{\dagger}$. The space of test functions is given by $\mathcal{V}=\left\{\boldsymbol{v} \in \mathcal{U}|\boldsymbol{v}|_{\Gamma_{u}}=0\right\}$. We omit further details in the nature of the spaces $\mathcal{U}, \mathcal{V}$ and $\mathcal{Q}$, which can be found for instance in [12].

\subsection{Mixed finite element discretisation}

After introducing a suitable finite element discretisation of the domain $\Omega$ into $n_{e l}$ elemental domains $\Omega^{e}, e=1, \ldots, n_{e l}$, the discrete version of problem (1) can be stated as follows: Find $\boldsymbol{u}^{h} \in \mathcal{U}^{h}$ and $p^{h} \in \mathcal{Q}^{h}$ such that,

$$
\begin{aligned}
a\left(\boldsymbol{u}^{h}, \boldsymbol{v}^{h}\right)-b\left(p^{h}, \boldsymbol{v}^{h}\right) & =\left(\boldsymbol{g}, \boldsymbol{v}^{h}\right), & \forall \boldsymbol{v}^{h} \in \mathcal{V}^{h} \\
b\left(q^{h}, \boldsymbol{u}^{h}\right) & =0, & \forall q^{h} \in \mathcal{Q}^{h} .
\end{aligned}
$$

The existence, uniqueness and stability of the discrete problem is provided by the (i) coercivity of the operator $a\left(\boldsymbol{u}^{h}, \boldsymbol{v}^{h}\right)$ on $k e r \mathcal{Q}^{h}$, and (ii) the LBB or inf-sup condition of the discrete spaces $\mathcal{V}^{h}$ and $\mathcal{Q}^{h}[12]$. A large amount of attention has been dedicated in the literature for the construction of suitable LBB-compliant spaces (see for instance $[7,12,17,20]$ ), or methods that stabilise the solution $[24,16]$. When using non-compliant LBB mixed finite elements discretisations, a lack of convergence of the pressure field is observed. Alternatively, in penalty methods, these finite element interpolations suffer a locking phenomenon of the displacement field as the penalty parameter increases [12, 24].

We will resort next to a finite element interpolation of the spaces $\mathcal{V}$ and $\mathcal{Q}$ by using a set of

\footnotetext{
${ }^{\dagger}$ We denote with bold font space functions where each component of the elements of the space belong to the associated space function with non-bold font.
} 
standard Lagrangian polynomials as follows: $\boldsymbol{u}^{h}=I^{i} \boldsymbol{u}_{i}$ and $p^{h}=h^{j} p_{j}$, where $I^{i}$ and $h^{j}$ are the (nodal) displacement and pressure trial functions. The vectors $\boldsymbol{u}_{i}$ are the nodal displacements, and $p_{j}$ are elemental pressure values computed at certain interior points of each element $e$. Henceforth, we will assume that our discretised domain has $n_{\text {nodes }}$ nodes and $n_{p}$ pressure dof. The test functions $\boldsymbol{v} \in \mathcal{V}$ are discretised in the same way as the displacements $\boldsymbol{u} \in \mathcal{U}$. Using the mentioned interpolation, the discretised version of the equilibrium equations in (2) leads to the following system of equations [18]:

$$
\begin{aligned}
\mathbf{K u}-\mathbf{B}^{\mathrm{T}} \mathbf{p}=\mathbf{g} \\
\mathbf{B u}=\mathbf{0} .
\end{aligned}
$$

The vectors $\mathbf{u}$ contains all the nodal displacements $\boldsymbol{u}_{i}, i=1, \ldots, n_{\text {nodes }}$. Vector $\mathbf{p}$ contains the elemental pressure degrees of freedom $p_{j}, j=1, \ldots, n_{p}$. Matrix $\mathbf{K}$ is the standard Jacobian constructed by assembling the elemental matrices $\mathbf{k}_{e}^{i j}=2 \mu \int_{\Omega^{e}}\left(\nabla I^{i} \cdot \nabla I^{j}\right) \mathbf{I} d \Omega-\frac{2 \mu}{3} \int_{\Omega} \nabla I^{i} \otimes$ $\nabla I^{j} d \Omega$ corresponding to the coupling of nodes $i$ and $j$, and the load vector $\mathbf{g}$ is the assembly of the nodal contributions $\mathbf{g}^{i}=\int_{\Omega^{e}} I^{i} \boldsymbol{g} d \Omega$ for each elemental domain $\Omega^{e}$. On the other hand, the block $i j$ of the gradient operator matrix $\mathbf{B}$ associated to pressure dof $i$ and node $j$ can be expressed as,

$$
\mathbf{B}^{i j}= \begin{cases}\int_{\Omega} h^{i}\left(\nabla I^{j}\right)^{\mathrm{T}} d \Omega & , \text { pressure dof } i \text { and node } j \text { are in the same element } \\ \mathbf{0} & , \text { otherwise }\end{cases}
$$

where $\nabla I^{j}$ denotes the gradient of the shape function $I^{j}$ of node $j$. If we use a space $\mathcal{Q}^{h}$ of piecewise constant functions (like in the Q1P0 quadrilateral), the equation $\mathbf{B u}=\mathbf{0}$ is 
equivalent to the satisfaction of the $n_{p}=n_{e l}$ equations that impose the elemental conservation of volumes $\Omega^{e}$ :

$$
\int_{\Omega^{e}} \operatorname{div}\left(\boldsymbol{u}^{e}\right) d \Omega^{e}=\boldsymbol{u}_{j}^{e} \cdot \int_{\Omega^{e}} \nabla I^{j} d \Omega^{e}=\mathbf{b}_{e}^{j} \cdot \boldsymbol{u}_{j}^{e}=0, \forall e=1, \ldots, n_{e l},
$$

where only summation over the $j$ nodes for each element $e$ is understood. In our approach though, the pressure field can be of any order, and we will develop a solution method of (3a) for general mixed finite element interpolation, with a piecewise continuous pressure.

In the optimisation context, it can be shown that the equilibrium equations (3) are the Karush-Kuhn-Tucker (KKT) conditions of a constrained minimisation problem (or saddle point problem), which can be stated as follows [32]:

$$
\left\{\begin{array}{l}
\min _{\boldsymbol{u}^{h} \in U^{h}} \frac{1}{2} a\left(\boldsymbol{u}^{h}, \boldsymbol{u}^{h}\right)-\left(\boldsymbol{g}, \boldsymbol{u}^{h}\right) \\
\text { s.t. } b\left(p^{h}, \boldsymbol{u}^{h}\right)=0 .
\end{array}\right.
$$

The hydrostatic pressures $p^{h}$ can be then interpreted as a field of Lagrange multipliers that impose the condition $\operatorname{div} \boldsymbol{u}^{h}=0$ weakly. In the next section we describe a method to solve the constrained system of equations (3) which circumvents the computation of the Lagrange multipliers (or the pressure variables).

\section{MASTER-SLAVE APPROACH FOR LINEAR ELASTICITY}

\subsection{General approach}

The solution of the constraint equations in (3) can be performed resorting to the so-called null space method $[8,32,9]$. It consists in finding a projection matrix, let's say $\mathbf{N}$, whose range is 
equal to $k e r \mathbf{B}$, i.e. $\operatorname{range} \mathbf{N}=k e r \mathbf{B}$. Such matrix will therefore satisfy the properties,

$$
\mathbf{N}^{\mathrm{T}} \mathbf{B}^{\mathrm{T}}=\mathbf{0}, \quad \mathbf{B N}=\mathbf{0} .
$$

In this way, we can project the discretised equilibrium equations in (3) onto range $\mathbf{N}$ by pre-multiplying equation (3a) by $\mathbf{N}^{\mathrm{T}}$, which leads, after using (5), to the following system of equations:

$$
\begin{array}{r}
\mathbf{N}^{\mathrm{T}}(\mathbf{K} \mathbf{u}-\mathbf{g})=\mathbf{0}, \\
\mathbf{B u}=\mathbf{0}
\end{array}
$$

As it will explained in the next paragraphs, the master-slave approach provides in fact a general way to obtain such matrix $\mathbf{N}$.

The algebraic equations $\mathbf{B u}=\mathbf{0}$ introduce a set of dependences among the discretised displacement variables. Therefore, and in parallel with other applications of the master-slave approach $[26,31,30,29]$, we can split the nodal displacement degrees of freedom $\mathbf{u}$ into a set of $n_{s}$ slave (dependent) displacements $\mathbf{u}_{s}$, and $n_{m}$ master (independent) displacements $\mathbf{u}_{m}$. The global vector of displacements is then expressible as $\mathbf{u}^{\mathrm{T}}=\left\{\mathbf{u}_{s}^{\mathrm{T}} \mathbf{u}_{m}^{\mathrm{T}}\right\}$, and the incompressible constraint equations $\mathbf{B u}=\mathbf{0}$ may be in turn partitioned as $\mathbf{B}=\left[\mathbf{B}_{s} \mathbf{B}_{m}\right]$. Accordingly, we can rewrite $\mathbf{B u}=\mathbf{0}$ as,

$$
\mathbf{B}_{s} \mathbf{u}_{s}+\mathbf{B}_{m} \mathbf{u}_{m}=\mathbf{0} .
$$

If the constraint equations in (7) are independent (we will discuss in Section 3.3 the case when they are not), matrix $\mathbf{B}_{s}$ is invertible and we may rewrite the dependence relationship 
as,

$$
\mathbf{u}_{s}=-\mathbf{B}_{s}^{-1} \mathbf{B}_{m} \mathbf{u}_{m}
$$

Thus, any displacement field with the form

$$
\mathbf{u}=\left\{\begin{array}{c}
-\mathbf{B}_{s}^{-1} \mathbf{B}_{m} \mathbf{u}_{m} \\
\mathbf{u}_{m}
\end{array}\right\}=\mathbf{N} \mathbf{u}_{m}
$$

will satisfy the constraint equation (3b), and hence, is a weakly solenoidal discretised field.

The master-slave transformation matrix $\mathbf{N}$ in (8a) is defined by,

$$
\mathbf{N}=\left[\begin{array}{c}
-\mathbf{B}_{s}^{-1} \mathbf{B}_{m} \\
\mathbf{I}
\end{array}\right]
$$

with $\mathbf{I}$ the $n_{m} \times n_{m}$ identity matrix. It can be verified that the columns of matrix $\mathbf{N}$ belong to $\operatorname{ker} \mathbf{B}$, and thus $\mathbf{B N}=\mathbf{0}$, as we wanted. It follows that matrix $\mathbf{N}$ may be used to project the equilibrium equations as shown in (6a), which together with relation (8a) leads to the following system of equations:

$$
\mathbf{N}^{\mathrm{T}} \mathbf{K N} \mathbf{u}_{m}-\mathbf{N}^{\mathrm{T}} \mathbf{g}=\mathbf{0} .
$$

The advantage of this system of equations with respect to the original system (3) is twofold:(i) the pressure unknowns $\mathbf{p}$ have been eliminated, and (ii) it contains $n_{s}$ displacement dof less than $\mathbf{u}$ in (3). Note also that if we compute our nodal displacements according to (8a), the constraint $\mathbf{B u}=\mathbf{0}$ will be always satisfied by construction, and thus, it does not need to be explicitly imposed. 
On the other hand, the elemental hydrostatic pressures can be computed from the first $n_{s}$ components of equation (3a). More explicitly, if we split the rows of the vectors $\mathbf{K u}$ and $\mathbf{g}$ according to the same partitioning used for $\mathbf{B}$, we may write them as,

$$
\mathbf{K u}=\left\{\begin{array}{c}
(\mathbf{K u})_{s} \\
(\mathbf{K u})_{m}
\end{array}\right\}, \quad \mathbf{g}=\left\{\begin{array}{c}
(\mathbf{g})_{s} \\
(\mathbf{g})_{m}
\end{array}\right\},
$$

where $(\mathbf{v})_{s} \in \mathbb{R}^{n_{s}}$ and $(\mathbf{v})_{m} \in \mathbb{R}^{n_{m}}$ denote the rows associated to the slave and master dof of a vector $\mathbf{v} \in \mathbb{R}^{n_{\text {dof }}}$, respectively. From the first $n_{s}$ equations of (3a) we can deduce the following expression for $\mathbf{p}$ :

$$
\mathbf{p}=\mathbf{B}_{s}^{-\mathrm{T}}(\mathbf{K u}-\mathbf{g})_{s} .
$$

It can be verified that this $\mathbf{p}$ satisfies as well the last $n_{m}$ equations of (3a), which can be written as

$$
(\mathbf{K u})_{m}-\mathbf{B}_{m}^{\mathrm{T}} \mathbf{p}=(\mathbf{g})_{m}
$$

Indeed, inserting the expression of $\mathbf{N}$ in (8b) into the reduced system of equilibrium equations $\mathbf{N}^{\mathrm{T}}(\mathbf{K u}-\mathbf{g})=\mathbf{0}$, we obtain the relation:

$$
\mathbf{B}_{m}^{\mathrm{T}} \mathbf{B}_{s}^{-\mathrm{T}}(\mathbf{K u}-\mathbf{g})_{s}=(\mathbf{K u}-\mathbf{g})_{m}
$$

If we replace $\mathbf{p}$ in (11) by its expression in (10), it can be observed that the previous equation and (11) are identical. 
We note that all the terms contained in (10) have been computed previously, or can be obtained in parallel with the computation of the term $\mathbf{B}_{s}^{-1} \mathbf{B}_{m}$ in matrix $\mathbf{N}$, which will be explained next.

\subsection{Implementation of the method}

The solution process given so far is well defined as far as $\mathbf{B}_{s}$ is non-singular. In our implementation of the code, we transform the gradient operator $\mathbf{B}=\left[\begin{array}{l}\mathbf{B}_{s} \\ \mathbf{B}_{m}\end{array}\right]$ into the matrix form $\left[\mathbf{I}-\mathbf{B}_{s}^{-1} \mathbf{B}_{m}\right]$ using a Gaussian decomposition process. This strategy permitted us to choose the slave degrees of freedom $\mathbf{u}_{s}$ as those that provided the maximum pivots, which minimises the lost of arithmetical precision in matrix $\mathbf{N}$. We note though that while the number of slave dof $n_{s}$ is independent of the chosen strategy, there are different suitable options for a selection of the actual partitioning of the dof. For instance, a plausible choice when sparseness is exploited may be to minimise the lost of sparseness of matrix $\mathbf{K}$ after the matrix product $\mathbf{N}^{\mathrm{T}} \mathbf{K N}$ is performed. A similar solution method in the context of linear analysis and using graph theory can be found in [1]. For the numerical examples we have tested, different strategies have always provided identical results.

The decomposition process and the matrix products in (9) are in fact the main additional computational costs introduced by the solution method. However, both operations are easily parallelisable. This fact, and the reduction of the number of variables in the system of equations (9), alleviate this additional cost.

For instance, it can be verified that for a $2 \mathrm{D}$ squared domain with $n_{e l}$ equal quadrilateral elements, the reduction in the system of equations approaches $50 \%$ and $62.5 \%$ for Q1P0 and Q2P1 interpolations, respectively, as $n_{e l}$ increases. However, the solution of $n_{m}$ systems 
of equations with dimensions $n_{s} \times n_{s}$, plus two matrix multiplications of $n_{m} \times\left(n_{s}+n_{m}\right)$ must be added. Since the number of operations of the Gaussian decomposition is equal to $[27] n_{s}^{3} / 3+n_{m} \times n_{s}^{2}-n_{s} / 3$, and the product $\mathbf{N}^{\mathrm{T}} \mathbf{K N}$ requires at least $2\left(n_{s}+n_{m}\right)^{2} n_{m}$ operations (without taking into account the sparseness of the matrices involved), it can be estimated that, for the mentioned problem and with equivalent displacement interpolations, the computational cost of the master-slave approach is at least four times larger. However, the additional operations can be easily parallelisable, as we have done in our implementation. Moreover, since we are exactly satisfying the discretised kinematic constraints, we show in Section 5, that for non-linear problems, coarser meshes within the master slave-approach lead to more accurate results than finer meshes using penalty methods.

The singularity of matrix $\mathbf{B}_{s}$, detected by the presence of maximum pivots which are equal (or very close) to 0 , is related to the stability conditions of the system in (3). The latter is in turn dependent on the nature of the finite element spaces employed for the displacement and pressure fields $[34,23]$. We show in the next section that whenever $\mathbf{B}_{s}$ is singular, a set of pressure modes arises which are associated to the rank-deficiency of $\mathbf{B}_{s}[34,7,24]$.

\subsection{Spurious pressure modes}

The stability and solvability conditions of the discretised problem in (3) are governed by the inf-sup condition, which has the following matrix expression $[5,11,12,7]$ :

$$
\inf _{0 \neq \mathbf{u}^{h} \in \mathcal{V}^{h}} \sup _{0 \neq \mathbf{q}^{h} \in \mathcal{Q}^{h}} \frac{\mathbf{q}^{\mathrm{T}} \mathbf{B u}}{\left\|\mathbf{u}^{h}\right\|_{\mathcal{V}^{h}}\left\|\mathbf{q}^{h}\right\|_{\mathcal{Q}}} \geq \beta>0
$$

with $\beta$ a parameter independent of the mesh size. If $\mathbf{B}_{s}$ is singular, we can find a vector $\mathbf{p}_{0} \neq \mathbf{0}$ such that $\mathbf{B}^{\mathrm{T}} \mathbf{p}_{0}=\mathbf{0}$, which nullifies the numerator in (12), and thus violates the inf-sup 
condition. In fact, $\operatorname{ker} \mathbf{B}^{\mathrm{T}}$ defines a set of pressure modes $\mathbf{p}_{0}$, whose spurious nature is revealed by noting that since $\mathbf{B}^{\mathrm{T}} \mathbf{p}_{0}=\mathbf{0}$, any solution of the form $\overline{\mathbf{p}}=\mathbf{p}+\mathbf{p}_{0}$ will also satisfy the equilibrium equations (3). The singularity of $\mathbf{B}_{s}$ can be interpreted as the underconstricton of the displacement field [24], which in many cases is a pathological byproduct of the numerical discretisation. The conditions under which such modes arise have been largely studied (see for instance [23] for a study of the chess-board modes in the 4-node quadrilateral element). However, for fully Dirichlet boundary conditions, a physically consistent constant pressure mode exists, which the solution method of the equilibrium equations should detect. For this reason, we will describe next a method to compute such potential pressure modes.

We will assume that equation $\mathbf{B u}=\mathbf{0}$ includes $n_{s}$ constraint equations, $r$ independent equations and $t=n_{s}-r>0$ dependent equations. Therefore, $\mathbf{B}$ is a $n_{s} \times n_{\text {dof }}$ matrix, where $n_{d o f}$ is total number of displacement degrees of freedom of the discretised domain, and $\operatorname{rank} \mathbf{B}=r<n_{s}$, i.e. $\mathbf{B}$ is rank-deficient. This can be regarded as the presence of dependent constraint conditions which do not impose further restrictions on the divergence-free displacement field $\mathbf{u}$ (imposing $r$ conditions already leads to a divergence-free displacements in a weak sense, for the space of pressures $Q^{h}$ considered). Thus, the constraint equation $\mathbf{B u}=\mathbf{0}$ contains $t=n_{s}-r$ dependent constraints which we will discard. The greater the rank-deficiency of $\mathbf{B}$ the greater the dimensions of $k e r \mathbf{B}^{\mathrm{T}}$, and thus the larger the number of pressure modes that satisfy $\mathbf{B}^{\mathrm{T}} \mathbf{p}_{0}=\mathbf{0}$.

This fact can be demonstrated algebraically by further partitioning matrix $\mathbf{B}_{s}$ into $r$ and $t$ rows with the linearly independent and dependent constraints, respectively, i.e. 


$$
\mathbf{B}=\left[\begin{array}{lll}
\mathbf{B}_{r r} & \mathbf{B}_{r t} & \mathbf{B}_{r m} \\
\mathbf{B}_{t r} & \mathbf{B}_{t t} & \mathbf{B}_{t m}
\end{array}\right]
$$

where $\mathbf{B}_{r r}$ is now an invertible $r \times r$ matrix. From the dependency of the last $t$ rows, matrix B accepts also the form:

$$
\mathbf{B}=\left[\begin{array}{ccc}
\mathbf{B}_{r r} & \mathbf{B}_{r t} & \mathbf{B}_{r m} \\
\mathbf{B}_{t r} & \mathbf{B}_{t r} \mathbf{B}_{r r}^{-1} \mathbf{B}_{r t} & \mathbf{B}_{t r} \mathbf{B}_{r r}^{-1} \mathbf{B}_{r m}
\end{array}\right]=\left[\begin{array}{cc}
\mathbf{I} & \mathbf{0} \\
\mathbf{0} & \mathbf{B}_{t r} \mathbf{B}_{r r}^{-1}
\end{array}\right]\left[\begin{array}{ccc}
\mathbf{B}_{r r} & \mathbf{B}_{r t} & \mathbf{B}_{r m} \\
\mathbf{B}_{r r} & \mathbf{B}_{r t} & \mathbf{B}_{r m}
\end{array}\right]
$$

The last expression clearly shows that the second block of $t$ rows in the equation $\mathbf{B u}=\mathbf{0}$ adds redundant constraints in the displacement field $\mathbf{u}$, and therefore can be discarded without altering the solution of the mixed problem. Consequently, when a singular $\mathbf{B}_{s}$ is detected in the Gaussian elimination process, the following reduced gradient operator and master-slave transformation matrix will be used:

$$
\overline{\mathbf{B}}=\left[\begin{array}{lll}
\mathbf{B}_{r r} & \mathbf{B}_{r t} & \mathbf{B}_{r m}
\end{array}\right] ; \overline{\mathbf{N}}=\left[\begin{array}{cc}
-\mathbf{B}_{r r}^{-1} \mathbf{B}_{r t} & -\mathbf{B}_{r r}^{-1} \mathbf{B}_{r m} \\
\mathbf{I} & \mathbf{0} \\
\mathbf{0} & \mathbf{I}
\end{array}\right] .
$$

It can be deduced that a vector of nodal hydrostatic pressures satisfying the equilibrium equation in (3a) is expressible as

$$
\mathbf{p}=\left\{\begin{array}{c}
\mathbf{B}_{r r}^{-\mathrm{T}}\left((\mathbf{K u})_{r}-\mathbf{g}_{r}\right) \\
\mathbf{0}
\end{array}\right\}
$$

which can be proved following the steps in Section 3.1. On the other hand, any vector with the form, 


$$
\mathbf{p}_{0}=\left\{\begin{array}{c}
-\mathbf{B}_{r r}^{-\mathrm{T}} \mathbf{B}_{t r}^{\mathrm{T}} \mathbf{p}_{t} \\
\mathbf{p}_{t}
\end{array}\right\}
$$

will satisfy the relation $\mathbf{B}^{\mathrm{T}} \mathbf{p}_{0}=\mathbf{0}$ (use last expression in (13)), and thus, will be a pressure mode. The vector $\mathbf{p}_{t}$, which is arbitrary and is $t$-dimensional, spans $\operatorname{ker} \mathbf{B}^{\mathrm{T}}$, i.e. the space of all pressure modes. Some numerical examples that exhibit pressure modes are shown in Section 5.

\section{MASTER-SLAVE APPROACH FOR HYPERELASTIC MATERIALS}

We extend the master-slave approach to non-linear problems with kinematic constraints, in particular to incompressible non-linear elasticity. We will first briefly introduce the key ingredients of non-linear elasticity, and then write the equations of an incompressible material as a constrained problem, onto which we apply similar techniques to those introduced in the previous section.

\subsection{Hyperelastic compressible material}

We will denote by $\Omega_{0} \subset \mathbb{R}^{n_{s d}}$ and $\Omega \subset \mathbb{R}^{n_{s d}}$ the undeformed and deformed configurations of an elastic body $\mathcal{B}$. Following standard notation [10, 21, 33], such a deformation may be mathematically described by the map $\phi(\mathbf{X}, t): \mathbb{R}^{3} \times \mathbb{R} \rightarrow \mathbb{R}^{3}$ of material points $\mathbf{X}$ onto the spatial points $\mathbf{x}=\phi(\mathbf{X}, t)$. We introduce the deformation gradient tensor as the tangent map $\mathbf{F}=\frac{\partial x}{\partial \boldsymbol{X}}$ and its determinant $J=\operatorname{det}(\mathbf{F})$. We will also use the right Cauchy-Green deformation tensor $\mathbf{C}=\mathbf{F}^{\mathrm{T}} \mathbf{F}$, its invariants $I_{C}=\operatorname{det}(\mathbf{C})=J^{2}, I I_{C}=\mathbf{C}: \mathbf{C}$ and $I I I_{C}=\operatorname{trace}(\mathbf{C})$, and a strain energy function which is assumed to depend exclusively on these invariants 
$\Psi\left(I_{C}, I I_{C}, I I I_{C}\right)$.

For simplicity, we assume that no body forces are applied, and therefore the local spatial equilibrium equation of body $\mathcal{B}$ in statics reads [10, 33]:

$$
\operatorname{div} \boldsymbol{\sigma}=\mathbf{0},
$$

where $\boldsymbol{\sigma}$ is the Cauchy stress tensor, related to the strain energy function through the relation

$$
\boldsymbol{\sigma}=2 J^{-1} \mathbf{F} \frac{\partial \Psi}{\partial \mathbf{C}} \mathbf{F}^{\mathrm{T}}
$$

The stress tensor $\boldsymbol{\sigma}$ can be understood as a function of the material points $\mathbf{X}$, i.e. $\boldsymbol{\sigma}(\mathbf{X})$, or alternatively a function of a displacement field $\boldsymbol{u}(\mathbf{X})=\mathbf{x}(\mathbf{X})-\mathbf{X}$, i.e. $\boldsymbol{\sigma}(\boldsymbol{u})$. We will hereafter refer to the latter case, although we will omit its argument for clarity.

The solution of the equilibrium equation consists in finding a displacement $\boldsymbol{u}$, such that satisfies (15) at all points of the domain $\Omega_{0}$. As it is customary, the associated weak form is obtained by multiplying (15) by a test function $\boldsymbol{v} \in \mathcal{V}$, integrating over the deformed domain and integrating by parts, which leads to: Find $\boldsymbol{u} \in \mathcal{U}$ such that

$$
\bar{a}(\boldsymbol{u}, \boldsymbol{v})=(\boldsymbol{g}, \boldsymbol{v}), \forall \boldsymbol{v} \in \mathcal{V},
$$

where $\bar{a}(\boldsymbol{u}, \boldsymbol{v})$ and $(\boldsymbol{g}, \boldsymbol{v})$ are given by

$$
\begin{aligned}
\bar{a}(\boldsymbol{u}, \boldsymbol{v}) & :=\int_{\Omega} \boldsymbol{\sigma}: \nabla_{x} \boldsymbol{v} d \Omega \\
(\boldsymbol{g}, \boldsymbol{v}) & :=\int_{\Gamma_{g}} \boldsymbol{g} \cdot \boldsymbol{v} d \Gamma
\end{aligned}
$$


and $\boldsymbol{g}$ is a field of surface forces acting on the boundary $\Gamma_{g}$ of $\mathcal{B}$.

The finite element discretisation of (17) can be derived resorting to the standard Lagrangian functions $I^{i}(\mathbf{X}), i=1, \ldots, n_{\text {nodes }}$ and interpolating the displacement field and test functions in the usual manner: $\boldsymbol{u}(\mathbf{X})^{h}=I^{i}(\mathbf{X}) \boldsymbol{u}_{i}$ and $\boldsymbol{v}(\mathbf{X})^{h}=I^{i}(\mathbf{X}) \boldsymbol{v}_{i}$, where $\boldsymbol{u}_{i}$ and $\boldsymbol{v}_{i}$ are a set of nodal values. From the arbitrariness of the nodal values of the test functions, the following non-linear equations are obtained:

$$
\mathbf{t}-\mathbf{f}=\mathbf{0},
$$

where the component associated to the dof of node $i$ of the elastic load vector $\mathbf{t}$ and the external load vector are given by $\mathbf{t}^{i}=\int_{\Omega} \boldsymbol{\sigma} \nabla_{x} I^{i}(\mathbf{X}) d \Omega$ and $\mathbf{g}^{i}=\int_{\Gamma_{g}} \boldsymbol{g} I^{i}(\mathbf{X}) d \Gamma$, respectively (see for instance [10]). We remark that for the usual strain energy functions, $\mathbf{t}$ will be in general non-linear, and thus $\mathbf{t} \neq \mathbf{K u}$ with $\mathbf{K}$ a constant matrix. Problem (18) can be solved by linearising it and resorting to the Newton-Raphson process, which at iteration $(k)$, leads to the following iterative scheme:

$$
\mathbf{K}^{(k)} \Delta \mathbf{u}=\mathbf{f}^{(k)}-\mathbf{t}^{(k)}
$$

Vector $\Delta \mathbf{u}^{(k+1)}$ are the set of nodal iterative displacements, and matrix $\mathbf{K}$ is the Jacobian of $\mathbf{t}$, i.e. $D \mathbf{t}[\Delta \mathbf{u}]=\mathbf{K} \Delta \mathbf{u}$.

\subsection{Hyperelastic incompressible material}

For an incompressible material, the following additional point-wise constraint must be satisfied: 


$$
J(\mathbf{X})=\operatorname{det} \mathbf{F}=1, \forall \mathbf{X} \in \Omega_{0} .
$$

Furthermore, in incompressible deformations, the right Cauchy-Green tensor $\mathbf{C}$ must also satisfy a kinematic condition [10], namely to be expressible as $\widehat{\mathbf{C}}=J^{-2 / 3} \mathbf{C}$. The modelling of incompressible hyperelastic materials requires thus to consider a version of the strain energy function $\widehat{\Psi}$ which depends on the modified invariants $I_{\widehat{C}}, I I_{\widehat{C}}$ and $I I I_{\widehat{C}}=1$, and additionally impose explicitly condition (20). We will next show that the structure of this constrained problem shares many similarities with the linear case.

We first note that due to the kinematic constraints, the Cauchy stress has a deviatoric component stemming from the modified strain energy function, according to (16), plus a hydrostatic part stemming from the kinematic constraint [10]:

$$
\boldsymbol{\sigma}=2 J^{-1} \mathbf{F} \frac{\partial \widehat{\Psi}}{\partial \mathbf{C}} \mathbf{F}^{\mathrm{T}}-p \mathbf{I}
$$

where the additional variable $p$ is the hydrostatic pressure. Consequently, for the present incompressible case, the form $\bar{a}$ in $(17 \mathrm{~b})$ can be rewritten as,

$$
\bar{a}(\boldsymbol{u}, p, \boldsymbol{v}):=\underbrace{\int_{\Omega} 2 J^{-1} \mathbf{F} \frac{\partial \widehat{\Psi}}{\partial \mathbf{C}} \mathbf{F}^{\mathrm{T}}: \nabla_{x} \boldsymbol{v} d \Omega}_{\widehat{a}(\boldsymbol{u}, \boldsymbol{v})}-\underbrace{\int_{\Omega} p \nabla_{x} \boldsymbol{v}: \mathbf{I} d \Omega}_{\widehat{b}(p, \boldsymbol{v})}
$$

Second, we will relax the point-wise constraint (20), and use its weak form, which reads:

$$
w(q, \boldsymbol{u}):=\int_{\Omega_{0}} q(J-1) d \Omega=0, \forall q \in Q,
$$


where as before, $q \in \mathcal{Q}$ can be regarded as a field of Lagrange multipliers. Combining equations (17), (22) and (23), we can formulate the constraint continuous problem of an hyperelastic material as: Find $(\boldsymbol{u}, p) \in \mathcal{U} \times \mathcal{Q}$ such that,

$$
\begin{aligned}
\widehat{a}(\boldsymbol{u}, \boldsymbol{v})-\widehat{b}(p, \boldsymbol{v})-(\boldsymbol{g}, \boldsymbol{v}) & =0, \forall \boldsymbol{v} \in \mathcal{V} \\
w(q, \boldsymbol{u}) & =0, \forall q \in \mathcal{Q} .
\end{aligned}
$$

The equations above have a similar structure as their linear counterparts (3) in Section 3. However, two main differences can be observed: (i) the gradient operator in $\widehat{a}(,$,$) and \widehat{b}($,$) are$ performed with respect to the spatial variables $\boldsymbol{x}$, and thus equation (24a) is non-linear, and (ii) equation (24b) is a non-linear constraint. We will deal with this non-linearities in the next section.

It can be verified that the weak form (24) are the KKT conditions of the minimisation of the total energy $\int_{\Omega} \widehat{\Psi} d \Omega-(\boldsymbol{g}, \boldsymbol{u})$, subjected to the incompressibility constraint $w(q, \boldsymbol{u})=0$ (in contrast to linear case, the total elastic energy can not be written as $\left.\frac{1}{2} \widehat{a}(\boldsymbol{u}, \boldsymbol{u})\right)$. The existence and requirements on $\mathcal{V}, \mathcal{U}$ and $\mathbf{F}$ for the existence of a solution to problem (24) have been analysed for instance in [35].

\subsection{Mixed finite element discretisation}

For reasons that will be clear below, we will not impose (23) directly, but its linearised form $D_{v} w(q, \boldsymbol{v})[\delta \boldsymbol{u}]$, which after using relation $D J[\delta \boldsymbol{u}]=J \nabla_{x} \delta \boldsymbol{u}:$ I yields:

$$
\widehat{b}(q, \delta \boldsymbol{u})=0 \forall q \in Q .
$$

with $\widehat{b}($,$) defined in (22).$ 
After discretising the fields $\boldsymbol{u}, \delta \boldsymbol{u}, \boldsymbol{v}, p$ and $q$ with the usual standard Lagrangian functions, the continuous weak form in (24) leads to the following non-linear equations:

$$
\begin{aligned}
\widehat{\mathbf{t}}-\mathbf{B}^{\mathrm{T}} \mathbf{p}-\mathbf{f} & =\mathbf{0} \\
\mathbf{B} \Delta \mathbf{u} & =\mathbf{0},
\end{aligned}
$$

where we have replaced the variation $\delta \boldsymbol{u}$ by the iterative displacement $\Delta \boldsymbol{u}$, i.e. its algorithmic counterpart. The global vector $\mathbf{p}$ contains all the nodal values of the hydrostatic pressures, i.e. $\mathbf{p}=\left\{p_{1} \ldots p_{n_{p}}\right\}^{\mathrm{T}}$, and the block $i j$ of the gradient operator matrix $\mathbf{B}$ associated to pressure dof $i$ and node $j$ is now given by

$$
\mathbf{B}^{i j}= \begin{cases}\int_{\Omega} h^{i} \nabla_{x} I^{j} d \Omega & , \text { pressure dof } i \text { and node } j \text { are in the same element } \\ \mathbf{0} & \text {, otherwise. }\end{cases}
$$

The component corresponding to node $i$ of the elastic and external load vectors $\widehat{\mathbf{t}}$ and $\mathbf{f}$ have the following expressions:

$$
\begin{aligned}
\widehat{\mathbf{t}}^{i} & =\int_{\Omega} 2 J^{-1} \mathbf{F} \frac{\partial \widehat{\Phi}}{\partial \mathbf{C}} \mathbf{F}^{\mathrm{T}} \nabla_{x} I^{i} d \Omega, \\
\mathbf{f}^{i} & =\int_{\Omega} I^{i} \boldsymbol{f} d \Omega .
\end{aligned}
$$

Equation (26) has a similar structure to equation (7), although for the current case $\mathbf{B}$ is non-linear and relates (infinitesimal) iterative displacements $\Delta \boldsymbol{u}$. 


\subsection{Master-slave approach}

Applying again the steps of the master-slave approach, we will split the vector $\Delta \mathbf{u}$ into a set of iterative slave displacements $\Delta \mathbf{u}_{s}$ and master iterative displacements $\Delta \mathbf{u}_{m}$. Partitioning the columns of $\mathbf{B}$ accordingly, i.e. $\mathbf{B}=\left[\begin{array}{ll}\mathbf{B}_{s} & \mathbf{B}_{m}\end{array}\right]$, we can construct a master-slave transformation matrix $\mathbf{N}$ such that:

$$
\Delta \mathbf{u}=\mathbf{N} \Delta \mathbf{u}_{m}
$$

with

$$
\mathbf{N}=\left[\begin{array}{c}
-\mathbf{B}_{s}^{-1} \mathbf{B}_{m} \\
\mathbf{I}
\end{array}\right]
$$

Like in the previous section, we can project the non-linear equation (26a) onto ker $\mathbf{B}$, which gives rise to the following non-linear equation:

$$
\mathbf{N}^{\mathrm{T}}(\widehat{\mathbf{t}}-\mathbf{f})=\mathbf{0}
$$

It is shown in the Appendix that the Jacobian of $\mathbf{N}^{\mathrm{T}} \widehat{\mathbf{t}}$ may be written as $\mathbf{N}^{\mathrm{T}}\left(\mathbf{K}_{E}-\mathbf{K}_{N}\right) \mathbf{N}$, where $\mathbf{K}_{E}$ is the standard Jacobian of the elastic load vector $\widehat{\mathbf{t}}$, and matrix $\mathbf{K}_{N}$ arises due to the linearisation of $\mathbf{N}$. Therefore, after using the iterative master-slave relation in (27), we obtain the following modified version of the Newton-Raphson process:

$$
\left(\mathbf{N}^{\mathrm{T}}\left(\mathbf{K}_{E}-\mathbf{K}_{N}\right) \mathbf{N}\right)^{(k)} \Delta \mathbf{u}_{m}=-\left(\mathbf{N}^{\mathrm{T}}(\widehat{\mathbf{t}}-\mathbf{f})\right)^{(k)}
$$


This system of equations has the minimal degrees of freedom and can be solved as an unconstrained problem with on the master iterative displacements $\Delta \boldsymbol{u}_{m}$.

The elemental pressures can be computed by using the first $n_{e l}$ equations in (26a), whence:

$$
\mathbf{p}=\mathbf{B}_{s}^{\mathrm{T}}(\widehat{\mathbf{t}}-\mathbf{f})_{s}
$$

We note that this step does not require any iterative process, but just the converged values of $\widehat{\mathbf{t}}$.

If we restrict our space $\mathcal{Q}^{h}$ to the set of piecewise constant functions, we are actually imposing a element-wise constraint, namely $\operatorname{vol}\left(\Omega^{e}\right)=\operatorname{vol}\left(\Omega_{0}^{e}\right)$, i.e.

$$
\int_{\Omega_{0}^{e}} \nabla_{x} \boldsymbol{u}: \mathbf{I} d \Omega=0, \quad e=1, \ldots, N_{e}
$$

We point out, that by imposing in (26b) the variation of the kinematic constraint, and not the constraint itself $w(q, \boldsymbol{u})=0 \forall q \in \mathcal{Q}$, a potential drift may exist after successive update of the iterative displacements as $\Delta \mathbf{u}=\mathbf{N} \Delta \mathbf{u}_{m}$, leading to a slight violation of the constraint. In order to avoid this effect, during the update process, we have projected the solution onto the discretised version of the weak form of the constraint: $w\left(h^{j}, \mathbf{u}\right)=0, j=1, \ldots, n_{p}$. This projection, necessary due to the non-linearity of the kinematic constraint $J-1=0$, required in fact one or maximum two iterations during the update process, and did not affect the quadratic convergence of the Newton-Raphson solution process. More precisely, the satisfaction of $w(q, \boldsymbol{u})=0 \forall q \in \mathcal{Q}$ in (24b) is equivalent to the following discretised non-linear equations:

$$
\mathbf{w}(\mathbf{u})=\mathbf{0},
$$


with $\mathbf{w}(\mathbf{u})^{\mathrm{T}}=\left\{w^{1}, \ldots, w^{n_{p}}\right\}$ and $w^{j}=w\left(h^{j}, \mathbf{u}\right)$, as defined in (23). After solving the linear system of equations in $(29)$ at iteration $(k+1)$, the following iterative process is performed in order to satisfy $(30)$ :

$$
\mathbf{w}\left(\mathbf{u}^{(\ell)}\right)+\mathbf{B}_{s}^{(\ell)} \Delta \mathbf{u}_{s}=\mathbf{0}
$$

Note that during these last iterations, $\mathbf{u}_{m}$ are kept fixed (i.e. $\mathbf{u}_{m}^{(\ell)}=\mathbf{u}_{m}^{(k+1)}$ ), and only the slave variables $\mathbf{u}_{s}$ are updated according to $\mathbf{u}_{s}^{(\ell+1)}=\mathbf{u}_{s}^{(\ell)}+\Delta \mathbf{u}_{s}$. Alternatively, as suggested by a reviewer, the equilibirum and projection iterations can be embedded in a single solution procedure by solving simultaneously (28) and (30):

$$
\left\{\begin{array}{c}
\mathbf{N}^{\mathrm{T}}(\hat{\mathbf{t}}-\mathbf{f}) \\
\mathbf{w}(\mathbf{u})
\end{array}\right\}=\mathbf{0}
$$

Indeed, the application of the Newton-Raphson solution process to this whole system of $n_{\text {dof }}$ non-linear equations yields the following expression:

$$
\left[\begin{array}{cc}
\mathbf{0} & \mathbf{N}^{\mathrm{T}}\left(\mathbf{K}_{E}-\mathbf{K}_{N}\right) \mathbf{N} \\
\mathbf{B}_{s} & \mathbf{B}_{m}
\end{array}\right]^{(k)}\left\{\begin{array}{c}
\Delta \mathbf{u}_{s} \\
\Delta \mathbf{u}_{m}
\end{array}\right\}=-\left\{\begin{array}{c}
\mathbf{N}^{\mathrm{T}}(\hat{\mathbf{t}}-\mathbf{f}) \\
\mathbf{w}(\mathbf{u})
\end{array}\right\}
$$

The upper block of equations is exactly (29), while the second block, is an alternative update of $\Delta \mathbf{u}_{s}$

Like in the linear case, we have computed matrix $\mathbf{N}$ using a Gaussian decomposition method, and we have numerically checked that the choice of the slave dof has no effects in the resulting displacements. 
In our numerical examples, we have used a Neo-Hookean material, which has the following strain energy function [10]:

$$
\Psi=\frac{\mu}{2}(\operatorname{trace}(\mathbf{C})-3)-\mu \ln J+\frac{\lambda}{2}(\ln J)^{2} .
$$

After inserting the kinematic constraint $\operatorname{det} \mathbf{F}=\operatorname{det} \mathbf{C}=1$ in the previous function, the modified version of $\Psi$, which only takes into account the deviatoric deformations, reads:

$$
\widehat{\Psi}=\frac{\mu}{2}(\operatorname{trace}(\widehat{\mathbf{C}})-3) .
$$

\section{NUMERICAL EXAMPLES}

In this section we describe the results obtained with the master-slave approach in linear (example 5.1) and non-linear analysis (examples 5.2-5.4). We have tested the interpolations Q1P0 (quadrilateral with 4 velocity nodes and one internal pressure node) and Q2P1 (quadrilaterals with 9 velocity nodes and three internal pressure nodes in $2 \mathrm{D}$, or hexahedra with 27 velocity nodes and 4 internal pressure nodes in 3D).

\subsection{Linear elastic problem with analytical solution}

We reproduce here some well known stability results of mixed elements using the master-slave approach. Consider the squared domain $(x, y) \in[0,1]^{2}$ with the displacements fully prescribed at the whole boundary with $\boldsymbol{u}_{0}=\mathbf{0}$. After defining the function $\chi(\xi)=\xi^{4}-2 \xi^{3}+\xi^{2}$, the following body load is applied: 


$$
\boldsymbol{g}(x, y)=\mu\left\{\begin{array}{c}
\chi(x) \chi^{\prime \prime \prime}(y)+\chi^{\prime \prime}(x) \chi^{\prime}(y) \\
-\chi^{\prime \prime \prime}(x) \chi(y)-\chi^{\prime}(x) \chi^{\prime \prime}(y)
\end{array}\right\}+\left\{\begin{array}{c}
3(x-0.5)^{2} \\
0
\end{array}\right\}
$$

The material is incompressible with a Lamé constant $\mu=100$. It can be verified that the problem has the following analytical solution:

$$
\boldsymbol{u}(x, y)=\left\{\begin{array}{c}
\chi(x) \chi^{\prime}(y) \\
-\chi^{\prime}(x) \chi(y)
\end{array}\right\} \quad, \quad p(x, y)=(x-0.5)^{3} .
$$

We have modelled this example with the master-slave approach and using two mixed finite element quadrilaterals: non LBB-compliant Q1P0 element (bilinear displacements and discontinuous constant pressures) and LBB-compliant Q2P1 element (biquadratic displacements and discontinuous linear pressures). The first element has been employed in a regular mesh and in a distorted one, shown in Figure 1a and 1b, respectively.

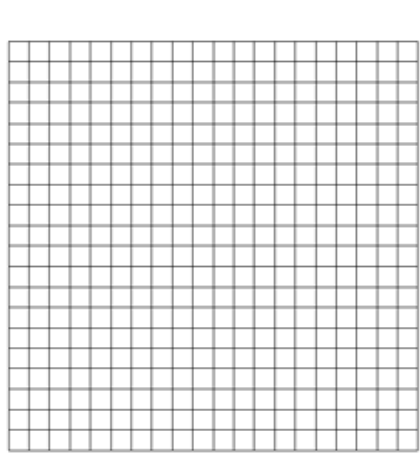

(a)



(b)

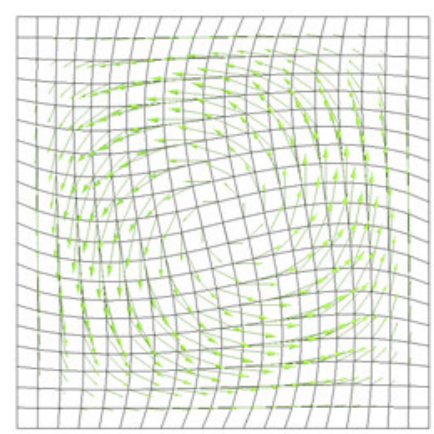

(c)

Figure 1. Regular mesh (a), distorted mesh (b), and displacements on the regular mesh using 400 Q1P0 elements in the linear problem.

While the element Q2P1, and the element Q1P0 in the distorted mesh gives only the 
(physically consistent) constant pressure mode, the Q1P0 in the regular mesh yields 2 popular spurious chess-board modes [7, 23], as shown in Figure 2b.

Figure 3 shows that all the elements have an order of convergence $k+1$ in the displacements for the $L_{2}$-norm, as expected [24]. However, regarding the pressure dof, while the Q2P1 element exhibits optimal convergence, the Q1P0 element yields a suboptimal convergence, regardless of the mesh employed.
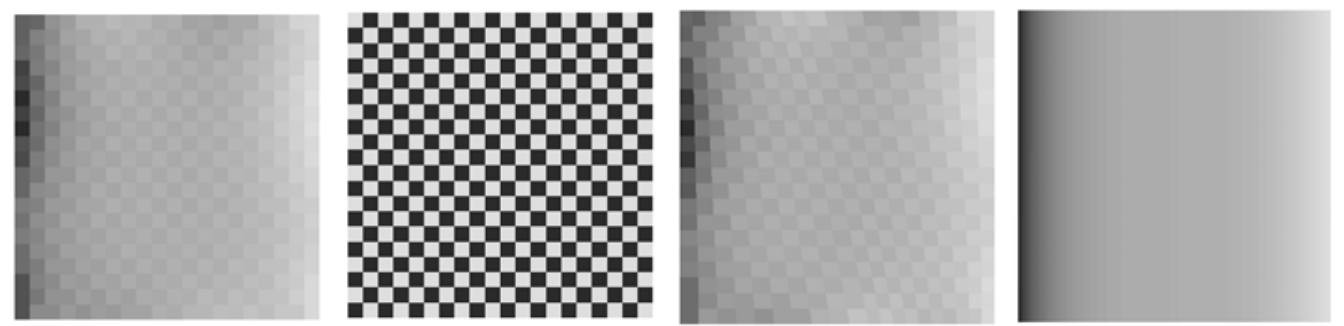

(a) Q1P0, hydrostatic (b) Q1P0, spurious pres- (c) Q1P0, hydrostatic (d) Q2P1, hydrostatic pressure, regular mesh sure mode, regular mesh pressure, distorted mesh pressure, regular mesh

Figure 2. Contour plots of the hydrostatic pressures $(\mathrm{a}, \mathrm{c}, \mathrm{d})$ and one of the two spurious pressure modes for the Q1P0 element on the regular mesh (b).The four plots use 400 elements.

\subsection{Non-linear elastic square with fully Dirichlet boundary conditions}

A model with the same geometry as in the previous example has been used. However, the material is now considered hyperelastic, and the boundary of the domain is subjected to a constant horizontal prescribed displacement $\boldsymbol{u}_{0}$ (see Figure 4). The solution is the displacement $\boldsymbol{u}_{0}$ throughout the domain, with a zero hydrostatic pressure and deviatoric stress, and a constant hydrostatic pressure mode. The aim of the example is to analyse the behaviour of the same elements studied before in a non-linear context. 


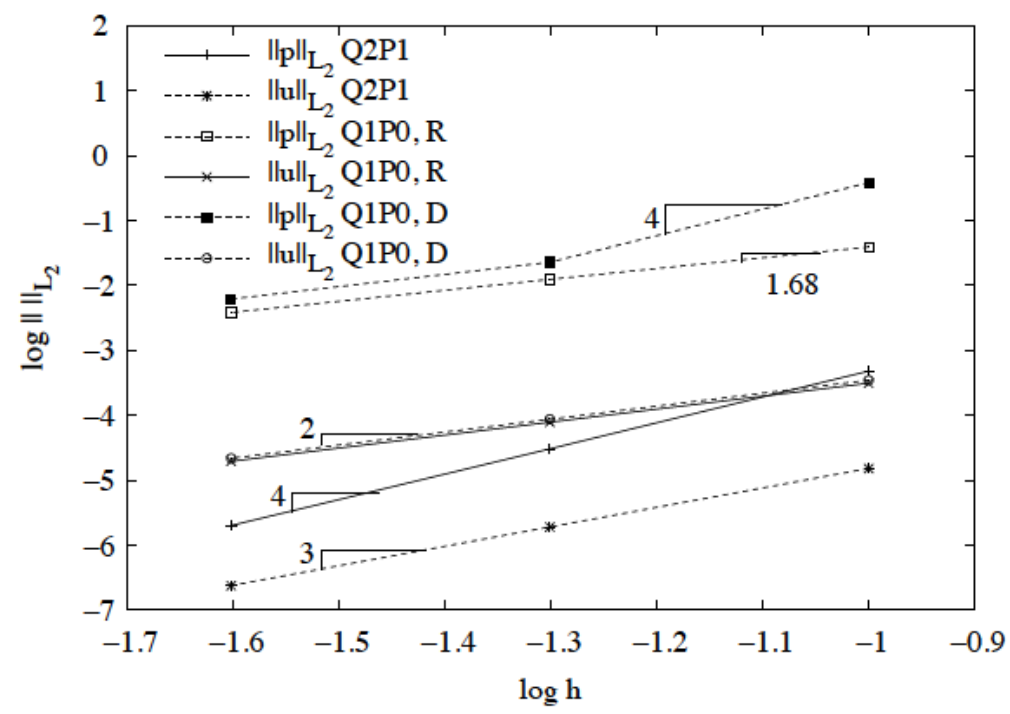

Figure 3. Pressure and displacement $L_{2}$-norm of the pressure and displacement errors. Regular and distorted meshes in the Q1P0 element are denoted by $\mathrm{R}$ and $\mathrm{D}$ respectively.

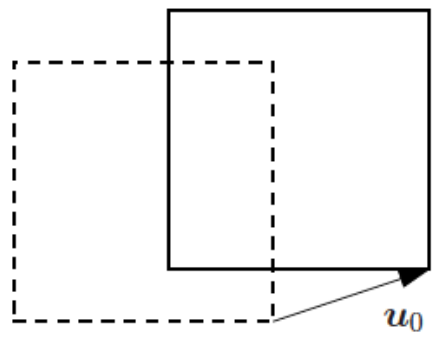

Figure 4. Non-linear square with fully Dirichlet boundary conditions

Since the solution of the non-linear problem is obtained by linearising successive iterative configurations, we will expect to encounter the same behaviour observed in the previous example. Indeed, when using the Q1P0 element, the first iterations of the Newton-Raphson process are performed onto a distorted mesh, but as the displacements approach the solution, 
the mesh becomes more and more regular. The Gauss decomposition then detects an additional nearly zero pivot, and thus the presence of the chess-board spurious modes, which form part of the final converged solution when using the Q1P0 element.

Both elements, Q1P0 and Q2P1 lead to the correct solution: zero hydrostatic pressure and deviatoric stress, and constant nodal displacements $\boldsymbol{u}_{0}$. In all cases, the Newton-Raphson process was stopped when the residual norm was smaller than $10^{-10}$, which results in an error in pressures and displacements smaller than $10^{-12}$ for the $L_{2}$-norm. However, as Table I shows, the mentioned mesh dependent response of the Q1P0 element introduced difficulties in the convergence. Nonetheless, and in spite of the resulting chess-board modes, the correct solution of the displacements was obtained.

The same test was also performed in 3D using three-dimensional mesh of $4 \times 4 \times 4$ regular hexahedra, and resorting to Q1P0 and Q2P1 mixed finite elements. As expected, the former interpolation lead to 11 spurious pressure modes, with similar chess-board patterns [12], whereas the latter yield only the constant pressure mode.

\subsection{Inflation of thick-walled cylinder}

We model the inflation of a thick-walled cylinder with initial internal and external radius $R_{1}=1.0$ and $R_{2}=1.25$ (see Figure 5), and Lamé constant $\mu=1$. Their deformed counterparts are denoted by $r_{1}$ and $r_{2}$. The analytical solution $[19,33]$ can be obtained from the symmetry condition, which leads to the following relation:

$$
r=\sqrt{r_{1}^{2}+R^{2}-R_{1}^{2}},
$$

where $R$ and $r$ are the undeformed and deformed radius of any point $R_{0} \leq R \leq R_{1}$. Also, 


\begin{tabular}{ccccc}
\hline Iter. & Q1P0, Res.Norm & Q1P0, \# Pres. modes & Q2P1, Res.Norm & Q2P1, \# Pres. modes \\
\hline 1 & $1.214 \mathrm{E}+01$ & 1 & $4.249 \mathrm{E}+00$ & 1 \\
2 & $1.764 \mathrm{E}+01$ & 1 & $5.634 \mathrm{E}+00$ & 1 \\
3 & $1.001 \mathrm{E}-03$ & 1 & $4.306 \mathrm{E}+00$ & 1 \\
4 & $7.210 \mathrm{E}+00$ & 1 & $1.317 \mathrm{E}-04$ & 1 \\
5 & $3.934 \mathrm{E}-04$ & 1 & $1.682 \mathrm{E}-08$ & 1 \\
6 & $8.904 \mathrm{E}+00$ & 1 & $4.216 \mathrm{E}-15$ & - \\
7 & $3.299 \mathrm{E}-05$ & 2 & - & - \\
8 & $4.146 \mathrm{E}-07$ & 2 & - & - \\
9 & $6.711 \mathrm{E}-12$ & 2 & - & \\
\hline
\end{tabular}

Table I. Residual Norm of the Newton-Raphson iterative process when using Q1P0 and Q2P1 elements in the non-linear elastic square problem

from the condition $\operatorname{det} \mathbf{F}=1$, we have that the only non-zero components of the deformation gradient $\mathbf{F}$ and the left Cauchy-Green strain tensor $\mathbf{b}=\mathbf{F F}^{\mathrm{T}}$ are $b_{r r}=\left(F_{r r}\right)^{2}=\left(\frac{R}{r}\right)^{2}$, $b_{\theta \theta}=\left(F_{\theta \theta}\right)^{2}=1 / b_{r r}, b_{z z}=F_{z z}=1$. The resulting Cauchy stresses can be obtained by solving the first component of the equilibrium equation $\operatorname{div} \boldsymbol{\sigma}=\mathbf{0}$, which in cylindrical coordinates and in the present case reads:

$$
\frac{\partial}{\partial r} \sigma_{r r}=\frac{1}{r}\left(\sigma_{\theta \theta}-\sigma_{r r}\right)
$$

From the expression $\boldsymbol{\sigma}=\mu\left(\mathbf{b}-\frac{1}{3} \mathbf{I}\right)-p \mathbf{I}$, this equation can be integrated, leading to:

$$
\sigma_{r r}(R)=\mu \log \left(\frac{R}{r}\right)+\frac{\mu}{2}\left(\frac{R}{r}\right)^{2}+k
$$

where from the boundary condition $\sigma_{r r}\left(R_{2}\right)=0$, we deduce $k=-\mu \log \left(R_{2} / r_{2}\right)-$ $0.5 \mu\left(R_{2} / r_{2}\right)^{2}$. 


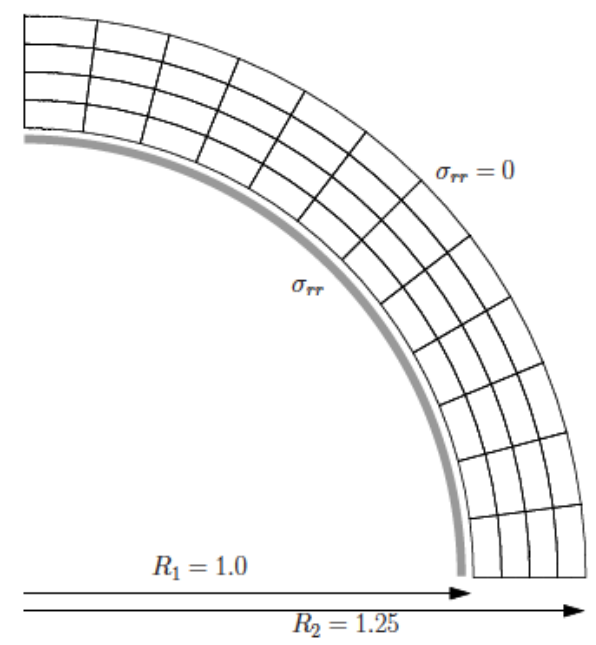

Figure 5. One quarter of the thick-walled cylinder using $4 \times 12$ elements

We have prescribed the internal radius $r_{1}$, and solved one quarter of the whole twodimensional cross-section. We have used the master-slave approach (MS), and compared the results with those obtained when employing the mean dilatation method (MD) [10], which uses a penalty parameter $k_{p}$ to impose the element-wise incompressibility constraint.

Figure 6 shows the evolution of the inflation pressure $p_{I}=-\sigma_{r r}\left(R_{1}\right)$. We have in fact computed the radial stress at the Gauss point that is closer to the inner surface $R=R_{1}$, and its corresponding analytical solution, which is a monotonically increasing function of $r_{1}$. Although the curves of the mean dilatation method approach the analytical solution for increasing values of the penalty parameter $k_{p}$, non of them is as close as the result of the master-slave approach. Figure 7 shows the $L_{2}$-norm of the error of the radial stresses and hydrostatic pressure. It can be observed that 4 radial elements in the master-slave approach yield a lower error than 8 radial elements in the mean dilatation method, with $k_{p}=1000$. In fact, the error in the latter method is reduced by increasing the penalty parameter, but 
not the number of elements. However, high values of $k_{p}$ are limited by the conditioning of the resulting system of equations. For this reason, the convergence tolerance of the residual in the Newton-Raphson process had to be reduced from $10^{-10}$ to $10^{-9}$ and $10^{-8}$, when $k_{p}=100$ and $k_{p}=1000$ was employed, respectively.

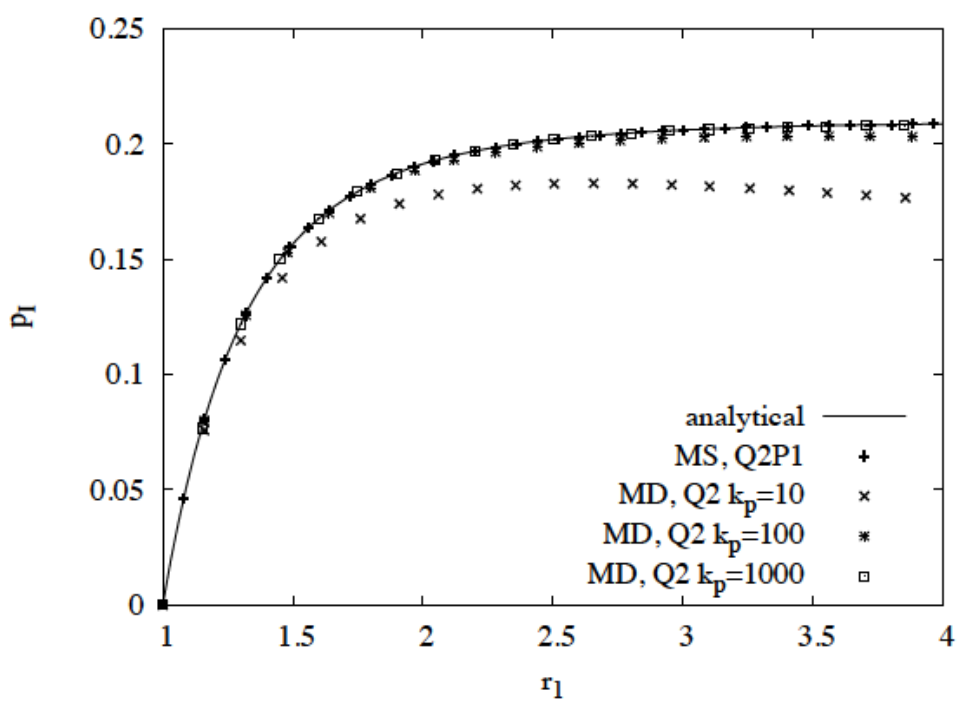

Figure 6. Evolution of the inflation pressure at the inner Gauss point using the master-slave approach

(MS) and the mean dilatation method (MD), with 4 elements in the radial direction.

Regarding the incompressibility constrain, Figure 8 plots the evolution of the relative volume error $\left|V-V_{0}\right| / V_{0}$, where $V$ is the volume of the cross-section. The error is in fact representative of the imposition of the weak form of the constraint. Again, in the mean dilatation method, the higher the values of $k_{p}$, the more accurate is the approximation of the constant volume constraint. In the master-slave approach, though, this constraint is satisfied exactly (to machine precision). We point out that the similarity of the stress and volume error underlines the importance to minimise the violation of the kinematic constraint. Apparently, when modelling incompressible materials and using penalty methods, errors in the kinematics induce errors in 


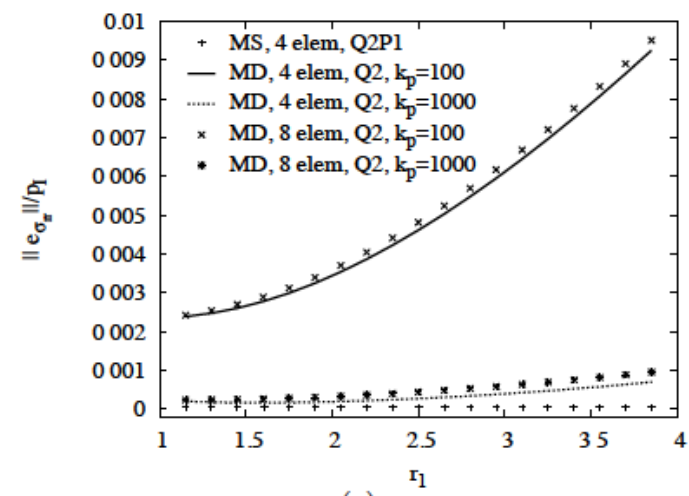

(a)

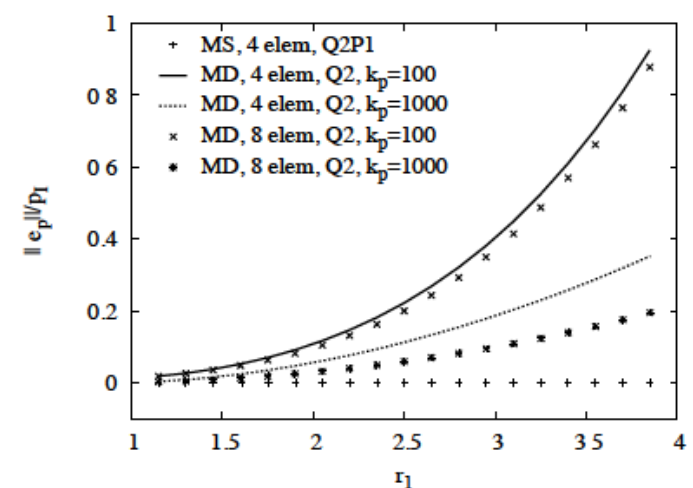

(b)

Figure 7. Relative error in $L_{2}$-norm of the radial stress (a) and the hydrostatic pressure (b) for the thick-walled cylinder. The number of elements indicated are those in the radial direction the stresses.

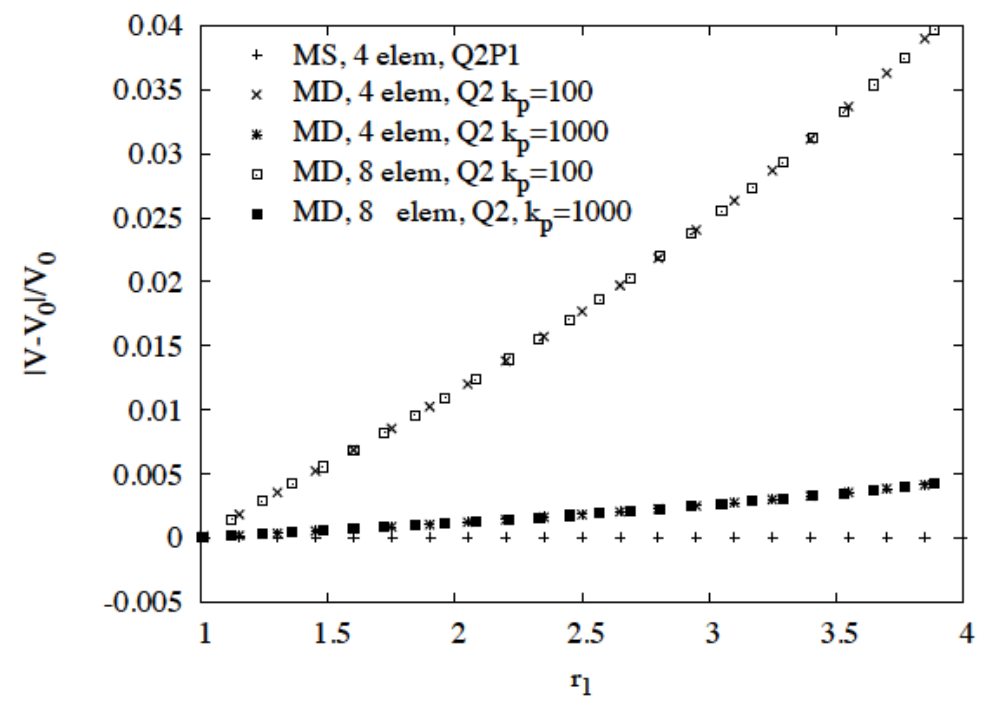

Figure 8. Evolution of the error of the total cross-section volume for the cylinder inflation problem.

Although no error estimates in non-linear analysis for the Q2P1 element could be found, the results shown for this example are in agreement with the numerical evidence described in 
[4], where robust results where obtained for this element.

\subsection{Inflation of thick-walled sphere}

The inflation of a thick walled sphere is a well studied problem, experimentally and analytically $[33,21]$. Although it is conceptually similar to the previous example, the solution leads to qualitatively different results. The dimensions of the inner and outer radius $R_{1}$ and $R_{2}$ are the same to those indicated in Figure 5 , and $\mu=1$ will be also used. The relation between the deformed and undeformed radius, taking into account the spherical symmetry and incompressibility condition is now given by,

$$
r=\left(r_{1}^{3}+R^{3}-R_{1}^{3}\right)^{1 / 3}
$$

The non-zero elements of the deformation gradient $\mathbf{F}$ and the left Cauchy-Green strain tensor $\mathbf{b}$ are $b_{r r}=\left(F_{r r}\right)^{2}=\frac{R^{2}}{r^{2}}, b_{\theta \theta}=\left(F_{\theta \theta}\right)^{2}=1 / \sqrt{b_{r r}}, b_{\phi \phi}=\left(F_{\phi \phi}\right)^{2}=b_{\theta \theta}$. The solution of this problem is obtained in the same manner as in the previous case. However, the first component of the equilibrium equation $\operatorname{div} \boldsymbol{\sigma}=\mathbf{0}$ in spherical coordinates reads:

$$
\frac{\partial}{\partial r} \sigma_{r r}=\mu \frac{2}{r}\left(\sigma_{\theta \theta}-\sigma_{r r}\right)
$$

which can be integrated, leading to the following inflation radial stress $\sigma_{r r}$ :

$$
\sigma_{r r}(R)=\left(2\left(\frac{R}{r}\right)+0.5\left(\frac{R}{r}\right)^{4}\right)+k
$$

The constant $k$ is obtained from the boundary condition $\sigma_{r r}\left(R_{2}\right)=0$, which yields $k=-\mu\left(2 R_{2} / r_{2}+0.5\left(R_{2} / r_{2}\right)^{4}\right)$. 
Figure 9 shows the initial mesh and the deformed sphere. In contrast to the previous example, the inflation pressure $p_{I}=-\sigma_{r r}\left(R_{1}\right)$ has a maximum, in the present case near the value $r_{1}=1.4922$. This is a popular effect experienced when inflating a balloon, which the numerical results in Figure 10 also capture. We note that more accurate and sophisticated models, such as the Ogden or Mooney-Rivlin [33, 21], can capture a more realistic behaviour, which is the increase of the internal pressure after the mentioned maximum. We have instead used the simple Neo-Hookean material model, which despite being physically inaccurate, it is sufficient to describe and study the computational problems encountered in incompressibility.

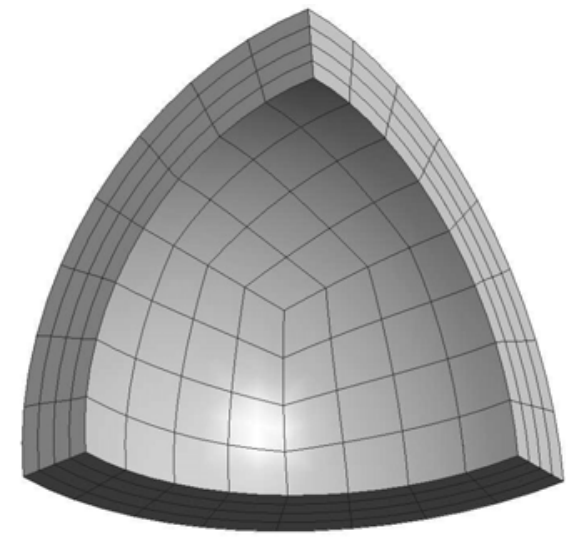

(a)

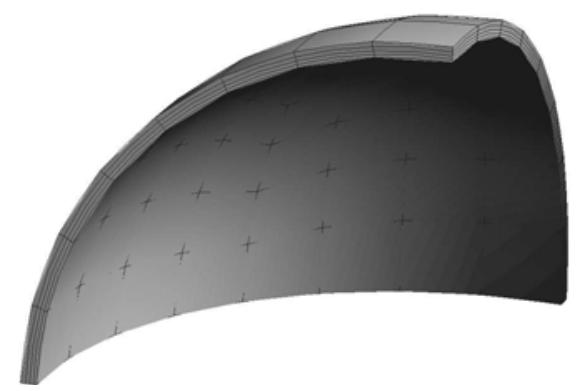

(b)

Figure 9. Initial mesh (a) and deformed configuration (b) of the sphere inflation problem

Like in the previous example, we have plotted the error in the radial stresses and the hydrostatic pressure (see Figures 11a and 11b). The error in the radial stress $\sigma_{r r}$ is for the master-slave approach higher than its two-dimensional counterpart. This anomaly is currently being investigated and could not be explained yet, although it is apparently not directly related to the incompressibility constraint. Nonetheless, the error in the hydrostatic pressure follows 


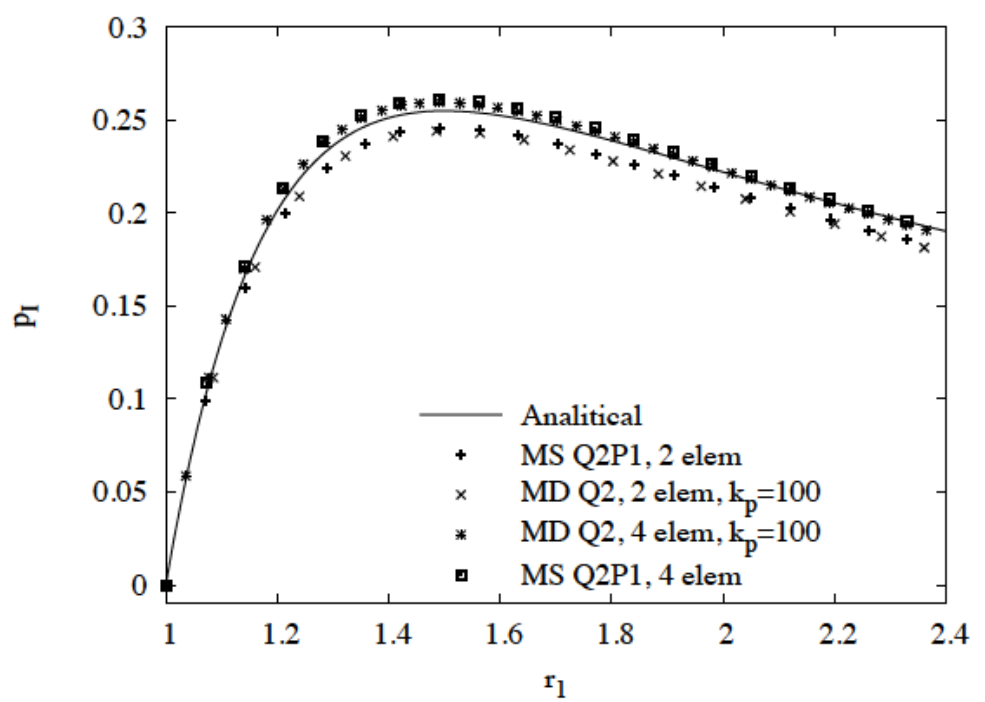

Figure 10. Evolution of the inflation pressure in the sphere problem. Comparison of master-slave approach (MS) and the mean dilatation method (MD)

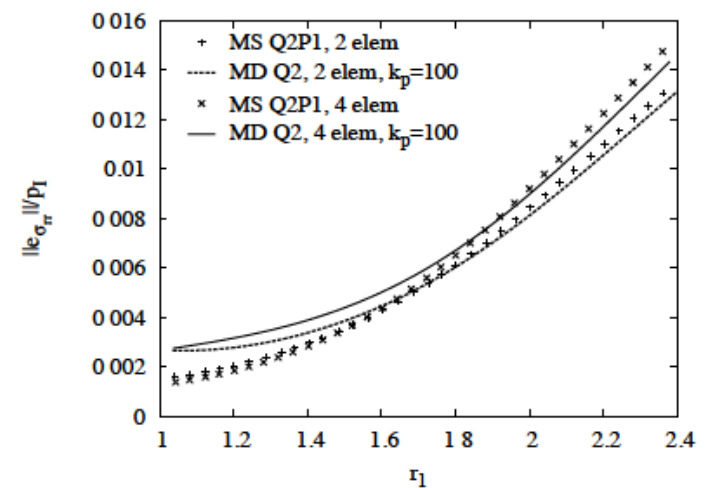

(a)

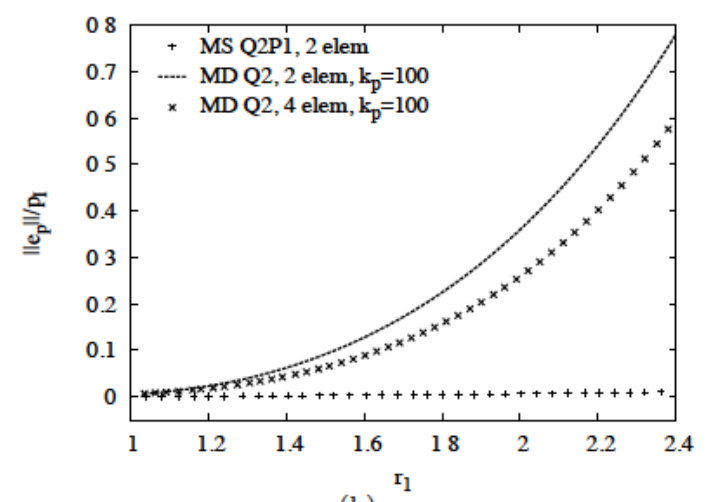

(b)

Figure 11. Relative error in $L_{2}$-norm in the radial stress (a) and the hydrostatic pressure (b) for the sphere inflation problem. Comparison of master-slave approach (MS) and the mean dilatation method 


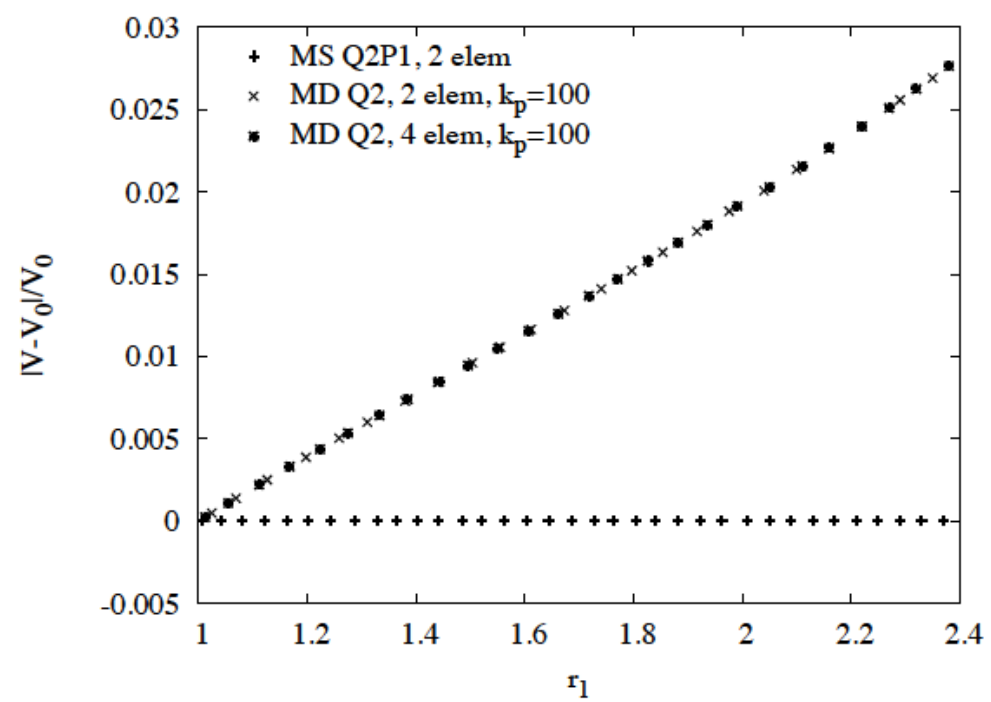

Figure 12. Evolution of the total volume error for the sphere inflation problem. Comparison masterslave approach (MS) and the mean dilatation method (MD)

the same trends as in the previous example, which is consistent with the volume error also plotted in Figure 12.

\section{CONCLUSIONS}

We have adapted to master-slave approach to construct divergence-free spaces of displacements and project the equilibrium equations of fully incompressible elasticity onto such space. In this way, a pure variational problem without constraints is solved, which has the minimum degrees of freedom. The technique developed here allows to detect the presence of pressure modes (physical or spurious) and determine their components in the space of discrete hydrostatic pressures. In fact, in non-linear analyses, the computation of the hydrostatic pressures is performed as a post-processing after the evaluation of the converged displacements have been obtained. 
By extending the master-slave approach to non-linear problems such as the modelling of hyperelastic materials, the incompressibility constraint is also satisfied in a weak sense. In the examples presented here, the satisfaction of this constraint appears also to determine the accuracy of the hydrostatic pressures.

We note that other basis of $k e r \mathbf{B}$ have been suggested in the literature, some of them with a physical representation (see for instance [20]). Here, we have introduced a general algebraic method to solve fully incompressible problems for arbitrary domains. The resulting formulation is based on a (weakly) divergence-free displacement field. However, the finite elements used are subjected to the same stability conditions as in other mixed formulations, namely the satisfaction of the LBB condition.

The projection of the equilibrium equations has a non-negligible computational cost: the inversion of a matrix, and two products of global matrices. This cost is however alleviated by the reduction in the number of variables in the final system of equations, and the fact that both operations can be easily parallelised, as we have done during our implementation. In addition, the use of LBB-compliant mixed element has been proved to lead accurate results in non-linear analysis.

With the present work, we have widen the applicability of the master-slave approach to other kind of constraints than those encountered in mechanisms and general contact mechanics. Although no reference to dynamic analyses has been made, we mention that the elimination of the Lagrange multipliers corresponding to the kinematic constraints is very advantageous in dynamics. Indeed, it has been shown that in linear dynamic analysis, infinite eigenvalues are associated to the Lagrange multipliers [15], and thus, by circumventing them, no differential-algebraic equation need to be solved. The application of the master-slave approach 
in incompressibility to non-linear dynamics remains yet unexplored.

\section{ACKNOWLEDGEMENT}

The author is financially supported by the Spanish research program Juan de la Cierva. This support is greatly acknowledged.

\section{APPENDIX}

\section{LINEARISATION OF NON-LINEAR EQUATIONS}

The set of non-linear equations is expressible as:

$$
\mathbf{N}^{\mathrm{T}} \widehat{\mathbf{t}}=\left[-\mathbf{B}_{m}^{\mathrm{T}} \mathbf{B}_{s}^{-\mathrm{T}} \mathbf{I}\right]\left\{\begin{array}{c}
\widehat{\mathbf{t}}_{s} \\
\widehat{\mathbf{t}}_{m}
\end{array}\right\} .
$$

The linear part can be written as the addition of two terms as follows:

$$
\Delta\left(\mathbf{N}^{\mathrm{T}} \widehat{\mathbf{t}}\right)=\mathbf{N}^{\mathrm{T}} \mathbf{K}_{E} \mathbf{N} \Delta \boldsymbol{x}_{m}+\left(\Delta \mathbf{N}^{\mathrm{T}}\right) \widehat{\mathbf{t}}
$$

where $\mathbf{K}_{E}$ is the standard Jacobian of the unconstrained element, and use of the relation $\Delta \mathbf{u}=\mathbf{N} \Delta \mathbf{u}_{m}$ has been done in the first term of the identity. By resorting to the relation $\Delta \mathbf{B}^{-\mathrm{T}}=-\mathbf{B}^{-\mathrm{T}}\left(\Delta \mathbf{B}^{\mathrm{T}}\right) \mathbf{B}^{-\mathrm{T}}$, the second term turns into,

$$
\left(\Delta \mathbf{N}^{\mathrm{T}}\right) \widehat{\mathbf{t}}=-\Delta\left(\mathbf{B}_{m}^{\mathrm{T}}\right) \mathbf{B}_{s}^{-\mathrm{T}} \widehat{\mathbf{t}}_{s}+\mathbf{B}_{m}^{\mathrm{T}} \mathbf{B}_{s}^{-\mathrm{T}}\left(\Delta \mathbf{B}_{s}^{\mathrm{T}}\right) \mathbf{B}_{s}^{-\mathrm{T}} \widehat{\mathbf{t}}_{s}=-\mathbf{N}^{\mathrm{T}}\left[\begin{array}{c}
\Delta \mathbf{B}_{s}^{\mathrm{T}} \\
\Delta \mathbf{B}_{m}^{\mathrm{T}}
\end{array}\right] \mathbf{B}_{s}^{-\mathrm{T}} \widehat{\mathbf{t}}_{s}
$$

We note here that the components of the product $\mathbf{B}_{s}^{-\mathrm{T}} \widehat{\mathbf{t}}_{s}$ are in fact the elemental pressures $\mathbf{p}$. In addition, from the definitions of matrices $\mathbf{B}_{s}$ and $\mathbf{B}_{m}$ in $(26 \mathrm{c})$, it can be verified that the product $\left[\begin{array}{ll}\mathbf{B}_{s} & \mathbf{B}_{m}\end{array}\right]^{\mathrm{T}} \mathbf{p}$ in the last term in (31) may be written as the assembling of elemental contributions, each one of which, denoted by $\boldsymbol{n}^{e}, e=1, \ldots, n_{e l}$, is expressible as, 


$$
\boldsymbol{n}^{e}=p_{k} \int_{\Omega^{e}} h^{k} J \nabla_{x} I^{i} d \Omega
$$

where summation on repeated index $k$ is understood ( $k$ is the number of pressure dof per element).

The elemental vector $\boldsymbol{n}^{e}$ has $n_{s d} \times n_{e n}$ dimensions, as the standard elemental displacement residual, where $n_{e n}$ is the number of nodes per element. The second term in (31) can be now written as,

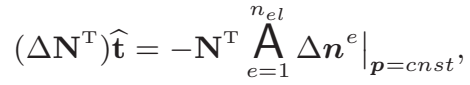

where $A$ denotes the standard assembling process. The linearisation of the elemental vectors $\boldsymbol{n}^{e}$ can be derived using the following formulae:

$$
\begin{aligned}
\Delta J & =J\left(\nabla_{x} I^{b} \cdot \Delta \mathbf{u}^{b}\right) \\
\Delta\left(\nabla_{x} I^{a}\right) & =\left(\Delta \mathbf{F}^{-\mathrm{T}}\right) \nabla_{X} I^{a}=-\mathbf{F}^{-\mathrm{T}} \Delta \mathbf{F}^{\mathrm{T}} \mathbf{F}^{-\mathrm{T}} \nabla_{X} I^{a}=-\mathbf{F}^{-\mathrm{T}}\left(\nabla_{X} I^{b} \otimes \Delta \boldsymbol{x}^{b}\right) \nabla_{x} I^{a} \\
& =-\left(\nabla_{x} I^{b} \otimes \nabla_{x} I^{a}\right) \Delta \mathbf{u}^{b} .
\end{aligned}
$$

It follows then that defining the following elemental stiffness matrix:

$$
\mathbf{k}_{a b}^{e}=p_{k} \int_{\Omega^{e}} h^{k}\left(\nabla_{x} I^{a} \otimes \nabla_{x} I^{b}-\nabla_{x} I^{b} \otimes \nabla_{x} I^{a}\right) J d \Omega^{e},
$$

and using again the relationship $\Delta \mathbf{u}=\mathbf{N} \Delta \mathbf{u}_{m}$, the linearisation of $\mathbf{N}^{\mathrm{T}} \widehat{\mathbf{t}}$ results in

$$
\Delta\left(\mathbf{N}^{\mathrm{T}} \widehat{\mathbf{t}}\right)=\mathbf{N}^{\mathrm{T}}\left(\mathbf{K}_{E}-\mathbf{K}_{N}\right) \mathbf{N} \Delta \mathbf{u}_{m}
$$

with $\mathbf{K}_{N}$ the assembling of the elemental matrices $\mathbf{k}_{a b}^{e}$ in (32). 
1. P Alotto and I Perugia. Tree-cotree implicit condensation in Magnetostatics. IEEE Trans. Magnetics, $36: 1523-1526,2000$.

2. D N Arnold, F Brezzi, and M Fortin. A stable finite element for the stokes equations. Calcolo, 21(4):223$344,1984$.

3. KJ Arrow, L Hurwicz, and H Uzawa. Studies in Linear and Nonlinear Programming. Standford University Press, Stanford, CA, 1958.

4. F Auricchio, L Beir ao da Veiga, C Lovadina, and A Reali. A stability study of some mixed finite elements for large deformatin elasticity problems. Comp. Meth. Appl. Mech. Engng., 194:1075-1092, 2005.

5. I Babuška. The finite element method with Lagrange multipliers. Numer. Math., 20:179-192, 1973.

6. I Babuška and R Narasimhan. The Babuška-Brezzi condition and the patch test: an example. Comp. Meth. Appl. Mech. Engng., 140:183-199, 1997.

7. KJ Bathe. Finite Element Procedures. Prentice Hall, 1996.

8. M Benzi, GH Golub, and J Liesen. Numerical solution of saddle point problems. Acta Numer., 14:1-137, 2005.

9. P Betsch. The discrete null space method for the energy consistent integration of constrained mechanical systems. Part I: Holonomic constraints. Comp. Meth. Appl. Mech. Engng., 194:5159-5190, 2005.

10. J Bonet and RD Wood. Non-linear continuum mechanics for finite element analysis. Cambridge University Press, 1997.

11. F Brezzi. On the existence, uniqueness and approximation of saddle-point problems arising from Lagrangian multipliers. Rev. Fr. Autom. Infor. Rech. Opér. Sér. Rouge, 8:129-151, 1974.

12. F Brezzi and M Fortin. Mixed and Hybrid Finite Element Methods. Springer-Verlag, 1991.

13. F Brezzi, M Fortin, and LD Marini. Error analysis of piecewise constant pressure approximations of Darcy's law. Comp. Meth. Appl. Mech. Engng., 195:1547-1559, 2006.

14. F Brezzi and J Pitkäranta. On the stabilization of the finite element approximations of the Stokes equations. In Efficient Solutions of Elliptic Systems, Proc. Gamm-Seminar, pages 11-19, Kiel, January 1984.

15. A Cardona and M A Géradin. Time integration of the equations of motion in mechanism analysis. Comput. Struct., 33(3):801-820, 1989.

16. R Codina. A stabilized finite element method for generalized stationary incompressible flows. Comp. Meth. Appl. Mech. Engng., 190:2681-2706, 2001.

17. M Crouzeix and P A Raviart. On the existence, uniqueness and approximation of saddle-point problems 
arising from Lagrangian multipliers. Rev. Fr. Autom. Infor. Rech. Opér. Sér. Rouge, 3:33-76, 1973.

18. J Donea and A Huerta. Finite Element Methods for Flow Problems. John Wiley \& Sons, 2003.

19. A E Green and W Zerna. Theoretical Elasticity. Oxford Univ. Press, 1963 [reprint 1st edition 1954, Oxford Clarendon Press].

20. D F Griffiths. An approximately diveregence-free 9-node velocity element (with variations) for incompressible flows. Int. J. Num. Meth. Fluids, 1:323-346, 1981.

21. G A Holzapfel. Nonlinear solid mechanics. A continuum approach for engineers. J Wiley \& Sons Ltd., 2000 .

22. U Hueck and H Schreyer. The use of orthogonal projections to handle constraints with applications to incompressible four-node quadrilateral elements. Int. J. Num. Meth. Engng., 35:1633-1661, 1992.

23. U Hueck, H Schreyer, and P Wriggers. On the incompressible constraint of the 4-node quadrilateral element. Int. J. Num. Meth. Engng., 38:3039-3035, 1995.

24. T J R Hughes. The finite element method. Linear static and dynamic finite element analysis. Dover Publ. Inc., 2000. Corrected reprint of the 1987 original [Prentice-Hall Int. Editions].

25. TJR Hughes, L P Franca, and M Balestra. A new finite element formulation for computational fluid dynamics: V. Circumventing the Babuška-brezzi condition: a stable Petrov-Galerkin formulation of the stokes problem accommodating equal-order interpolations. Comp. Meth. Appl. Mech. Engng., 59:85-99, 1986.

26. A Ibrahimbegović and S Mamouri. On rigid components and joint constraints in nonlinear dynamics of flexible multibody systems employing 3D geometrically exact beam model. Comp. Meth. Appl. Mech. Engng., 188:805-831, 2000

27. E Isaacson and H B Keller. Analysis of numerical methods. Dover publications, Inc, 1966.

28. G Jelenić and M A Crisfield. Non-linear master-slave relationships for joints in 3D beams with large rotations. Comp. Meth. Appl. Mech. Engng., 135:211-228, 1996.

29. G Jelenić and J J Muñoz. Multibody dynamics. Computational methods and applications. Ed: JC García Orden et. al. Computational Methods in Appl. Sc. Springer-Verlag, 2007.

30. J J Muñoz and G Jelenić. Sliding contact conditions using the master-slave approach with application on the geometracally non-linear beams. Int. J. Solids Struct., 41:6963-6992, 2004.

31. J J Muñoz, G Jelenić, and M Crisfield. Master-slave approach for the modelling of joints with dependent degrees of freedom in flexible mechanisms. Comm. Num. Meth. Engng., 19:689-702, 2003.

32. J Nocedal and S J Wright. Numerical Optimization. Springer Series in Operations Research. Springer- 
Verlag, 1999 .

33. RW Ogden. Non-linear elastic deformations, volume 26. Dover Publications, 1984.

34. RL Sani, PM Gresho, RL Lee, and DF Griffiths. The cause and cure (?) of the spurious pressures generated by certain FEM solutions of the incompressible Navier-Stokes equations: part 1. Int. J. Num. Meth. Fluids, 1:17-43, 1981.

35. P Le Tallec. Handbook of Numerical Analysis, vol III, chapter Numerical Methods for nonlinear threedimensional elasticity, pages 465-622. North Holland Amsterdam, 1994.

36. C Taylor and P Hood. A numerical sollution of the Navier-Stokes equations using the finite element technique. Comput. Fluids, 1:73-100, 1973. 